\title{
Optimization of Sensor and Wireless Network for Forest Fire Detection
}

\author{
Ganesh Sharma ${ }^{1}$ and Dipanjan Bhattacharjee ${ }^{2}$ \\ 12 Sikkim Manipal Institute of Technology, Majhitar, India \\ Igsharma.smit@gmail.com,22dipanjanbhattacharjee@yahoo.co.in
}

\begin{abstract}
Detection of forest fire is a complex and vital process. The system must be accurate and the response of the system must be fast. The main parameter to be measured in case of forest fire is temperature. Hence it is important to select appropriate temperature sensor considering the various parameters and real time scenario. In this paper selection of an appropriate temperature sensor for forest fire detection system is done based on real time experiment. Another important aspect of this system is the network for transmission of sensed data to base station for analysis. The network employed must be wireless and it must be secure, reliable, robust, easy to implement and cost effective. The loss of data or latency in the network can results in a disaster. AHP is used to select an appropriate network with higher throughput and lower latency.
\end{abstract}

Keywords: sensors, wireless networks, AHP, Zigbee, Wi-Fi, Bluetooth, DS18B20, MLX90614, BMP085

\section{Introduction}

Forest fire is one of the most often occurred phenomenon which claims loss of life, vegetation and property. Although there exists many fire detection systems but most of them are either not much accurate or the cost of implementation of such system are high. In such scenario the forest fire detection system based on WSN can be a reliable and cost effective solution to overcome above problem. Temperature is one of most important parameter that determines the occurrence of fire. Hence temperature sensor is vital in case of forest fire detection system. There are various types of temperature sensors available commercially but all of them are not suitable for temperature sensing in case of forest fire detection system. In case of forest fire detection system the sensor must be able to detect temperature without coming in contact with the burning object. Hence a real time experiment is required to select an appropriate temperature sensor. Another important system requirement for forest fire detection system is network. The wired network cannot be implemented in such system because it is not feasible due to geographical condition and higher cost of implementation. There are various types of wireless standards such as Wifi, Bluetooth, zigbee etc. There are various factors need to be considered, such as power consumption, transmission range, data rate and cost of implementation for selection of appropriate wireless standard for forest fire detection system. There are various methods used for selection of appropriate technology for any system considering various criteria and conditions. AHP is one of the processes used for selection of suitable product for any application. Here the best among three networks namely wifi, Bluetooth and zigbee are chosen based on criteria like data rate, range and power consumption with the help of AHP. 


\section{Literature Survey}

This paper describes about the forest fire detection system based on use of diode (4148) as temperature sensor and the wireless communication is achieved using GSM [2]. This paper basically describes about the advantage of zigbee over other WLAN technology like Bluetooth. It also states that other WLAN technology has larger power dissipation, shorter range, higher complexity compared to zigbee. It also highlights the use of zigbee in various low data rate application [8]. AHP can be used to select the trustworthy key management scheme in WSN. It conclude that AHP aided methods can prevent subjective judgement errors and increase the likelihood that the results are reliable. AHP aided methods provide useful insight into trade off embedded in decision making problems [10]. The detection of forest fire can be done using the sensor which can sense temperature and humidity such as RHT03. The wireless communication is achieved by the use of BTBee wireless sensor technology [6]. This paper describes the use of AHP for selecting the best temperature sensor. It describes the whole process of AHP for selecting the best sensor among various commercially available sensor based on various parameters to be taken under consideration [11]. The comparative study of various temperature sensors such as thermistor, LM35, RTD etc based on accuracy, response, range and application area is done. The conclusion was drawn that the decision on which temperature sensor to be used depend on range of temperature to be measured and application area [1]. Comparative performance analysis of six wireless protocols namely zigbee, Bluetooth, UWB, wifi, Wimax and GSM/GPRS is done. The conclusion is drawn that the selection of suitable protocol depends on parameters such as reliability, installation cost, security, data rate requirement etc [4]. The comparative study of wireless technology for industrial application is done. The study was done among zigbee, Bluetooth and wifi. The conclusion was made that zigbee is best suited for industrial application due to its low cost of deployment and its ability to function and survive in industrial setting like high RF noise floor, temperature extremes, rough handling etc [5]. The comparative study of zigbee, Bluetooth and wifi was done for its implementation in wireless fire alarm system. It was concluded that zigbee was preferred over wifi and Bluetooth for this application due to its lower power consumption which enhance the lifetime of sensor node [3].

\section{Selection of Temperature Sensor}

There are various temperature sensors available commercially. These sensors have different types of sensing technology and temperature sensing range. But all these sensors are not suitable for detecting temperature for forest fire detection system. For this purpose we have considered three temperature sensors namely BMP085, DS18B20 and MLX90614.

BMP085 is basically a barometric pressure sensor from Bosch Sensortec, but this device is also capable of measuring temperature along with pressure and altitude. This device can be connected to microcontroller board using I2C interface. It measures 16 bit uncompensated temperature. This raw data can be used to display the temperature in ${ }^{\circ} \mathrm{C}$ using the calibration data stored in its EEPROM. There are 11 calibration data of 16 bit each which makes total of 176 bits.

DS18B20 is a digital thermometer which provides 9 bits to 12 bits Celsius temperature measurement. It communicates with microcontroller board using 1 wire bus. Each DS18B20 has unique 64 bit serial number which allow multiple devices to function on same 1 wire bus.

MLX90614 is an infrared thermometer. This device can measure the temperature using the IR energy emitted by the object. This device consists of infrared thermopile detector (MLX81101) and signal conditioning ASSP (MLX90302) specially designed to process output of IR sensor. Both the above device is packed in single TO-39 package. There is an 
optical filter (long wave pass- 5.5 till $14 \mu \mathrm{m}$ ) that cut off the visible and near infrared radiation flux to provide ambient and sun light immunity.

Table 1. Comparison Table for Three Type of Temperature Sensor

\begin{tabular}{|c|c|c|c|}
\hline SPECIFICATION & BMP085 & DS18B20 & MLX90614 \\
\hline SUPPLY VOLTAGE & $1.8-3.6 \mathrm{~V}$ & $3-5.5 \mathrm{~V}$ & $3.6 \mathrm{~V}$ \\
\hline $\begin{array}{c}\text { STORAGE } \\
\text { TEMPERATURE }\end{array}$ & $-40^{\circ} \mathrm{C}-+85^{\circ} \mathrm{C}$ & $-55^{\circ} \mathrm{C}-+125^{\circ} \mathrm{C}$ & $-40^{\circ} \mathrm{C}-+125^{\circ} \mathrm{C}$ \\
\hline $\begin{array}{c}\text { OPERATING } \\
\text { TEMPERATURE }\end{array}$ & $-40^{\circ} \mathrm{C}-+85^{\circ} \mathrm{C}$ & $-55^{\circ} \mathrm{C}-+125^{\circ} \mathrm{C}$ & $-70^{\circ} \mathrm{C}-+380^{\circ} \mathrm{C}$ \\
\hline $\begin{array}{c}\text { INTERFACING } \\
\text { TECHNOLOGY }\end{array}$ & $12 \mathrm{C}$ & 1 WIRE BUS & SMBus \\
\hline ACCURACY & $\pm 1.0^{\circ} \mathrm{C}$ & $\pm 0.5^{\circ} \mathrm{C}$ & $\pm 0.5^{\circ} \mathrm{C}$ \\
\hline
\end{tabular}

\subsection{Problem Definition}

Above table shows the comparative study of three temperature sensors. These entire sensors have similar electrical characteristic and accuracy. The main problem is to find which sensor is suitable for application to detect the fire from distant by analyzing the rise in temperature. To select the appropriate sensor the real time experiment in presence of fire is done. In following section the experimental set up for real time experiment is described.

\subsection{Experimental Setup}

Figure 1 shows the circuit connection diagram to perform the experiment to select appropriate temperature sensor for detecting temperature for forest fire detection system.

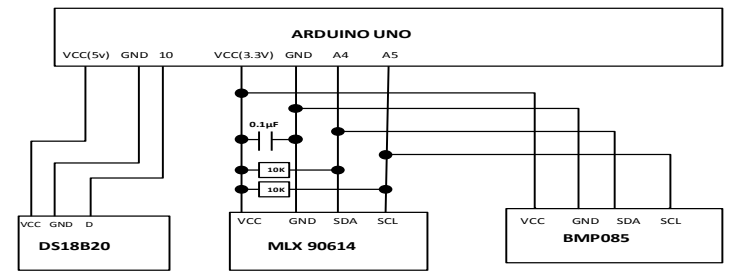

Figure 1. Connection Diagram of Three Sensors with Arduino Uno Board

Here two sensors MLX90614 and BMP085 are connected to the board using I2C interface. Although MLX90614 uses SMBus interface but it can be interfaced using I2C bus by using external pull up resistor of $10 \mathrm{~K}$ each on SDA and SCL line. The capacitor is connected between VCC and GND which act as filter to regulate DC supply for sensors. The implementation of filter makes the measurement accurate and stable. The supply voltage for above two sensor is 3.3V. DS18B20 is interface with the board by connecting data line of sensor to digital pin 10 of the board. The board is programmed such that it will print the output temperature of all three sensors in interval of 10 second in the serial monitor of the PC. 


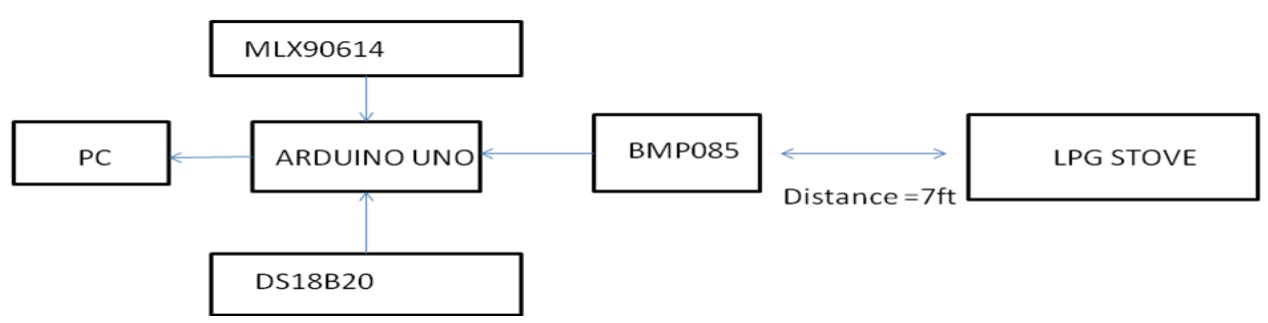

Figure 2. Set up for Experiment

All the sensors were connected to same arduino uno board. The board was connected to PC via USB cable. The output temperature of sensors was displayed in the serial monitor of the PC. The setup was placed at distance of $7 \mathrm{ft}$ from the gas stove. During the start of experiment BMP085 was showing temperature $19.5^{\circ} \mathrm{C}$, while DS18B20 was showing $18^{\circ} \mathrm{C}$ and MLX90614 was showing $18.4^{\circ} \mathrm{C}$. Initially stove was not ignited. After few minutes it was ignited and it was kept on for few minutes and then it was turned off. The readings of all three sensors were observable in the serial monitor of PC.

\subsection{Experimental Result}

The temperature reading of entire process was plotted based on the data available in the serial monitor.

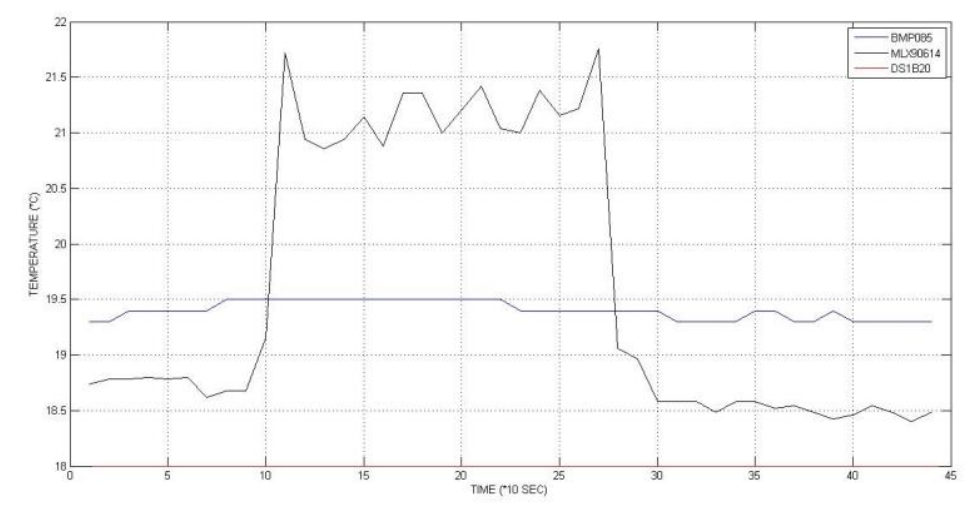

Figure 3. Output Temperature Plot of Three Sensors

From the above plot it is clearly visible that when the stove was ignited the temperature reading of DS18B20 and BMP085 didn't change. The increase in the temperature reading of MLX90614 can be observed when the stove was ignited and decrease in temperature reading when stove was turned off. The temperature sensor in case of forest fire detection system must be placed at an elevated height in order to protect them from flood and other damages. In such scenario the sensor must be able to detect the temperature from certain distance, hence from above result it can be concluded that in such scenario MLX90614 is suitable compared to other two sensors. MLX90614 is based on IR energy emitted by the body, hence it responds faster to change in temperature compared to other type of sensors.

\section{Selection of Wireless Network Based on AHP}

There are various wireless standards for use in forest fire detection system. Here we have considered three types of standard wifi, Bluetooth and zigbee. These entire standards are for PAN and LAN. These standard works in ISM band with frequency of $2.4 \mathrm{GHz}$.

Wifi is defined by the standard IEEE 802.11. It is set of low tier terrestrial network technology, operated in $2.4 \mathrm{GHz}$ and $5 \mathrm{GHz}$ ISM band. It has many variants like IEEE $802.11 \mathrm{a} / \mathrm{b} / \mathrm{g} / \mathrm{n}$. 
Bluetooth is defined by the standard IEEE802.15.1. It is low tier, terrestrial standard for short range communication. It is operated in three classes of devices. Class 1 has range of $100 \mathrm{~m}$, class 2 has range of $10 \mathrm{~m}$ and class 3 has range of $1 \mathrm{~m}$. Bluetooth and WLAN operate in same frequency band but they use different signalling to avoid interference.

Zigbee is defined by the standard IEEE 802.15.4. It is a low tier, terrestrial, Ad-hoc, wireless standard. It operates in three different bands. The three different bands are 2.4 GHZ, $868 \mathrm{MHZ}$ and $915 \mathrm{MHZ}$. In case of $2.4 \mathrm{GHZ}$ band the total spectrum is divided into 16 channels, in case of $915 \mathrm{MHZ}$ it is divided into 10 channels, whereas for 868 MHZ it has only 1 channel. It uses CSMA-CA for collision avoidance and DSSS for security and immunity to jamming. It uses various types of modulations such as PSK, QPSK, O-QPSK based on data rate requirement.

Table 2. Comparison Table for Three Wireless Technologies

\begin{tabular}{|c|c|c|c|}
\hline SPECIFICATION & $\begin{array}{c}\text { WI-FI } \\
(802.11)\end{array}$ & $\begin{array}{c}\text { BLUETOOTH } \\
(802.15 .1)\end{array}$ & $\begin{array}{c}\text { ZIGBEE } \\
(802.15 .4)\end{array}$ \\
\hline FREQUENCY BAND & $2.4 \mathrm{GHZ} / 5 \mathrm{GHZ}$ & $2.4 \mathrm{GHZ}$ & $\begin{array}{c}868 \mathrm{MHZ}, 941 \mathrm{MHZ} \\
82.4 \mathrm{GHZ}\end{array}$ \\
\hline RANGE & $250 \mathrm{M}$ & $20 \mathrm{M}$ & $1 \mathrm{KM}$ \\
\hline $\begin{array}{c}\text { MODULATION } \\
\text { SCHEME }\end{array}$ & $\begin{array}{c}\text { QAM, OFDM \& } \\
\text { ALAMOUTI }\end{array}$ & $\begin{array}{c}\text { PSK, FSK, SPREAD } \\
\text { SPECTRUM }\end{array}$ & $\begin{array}{c}\text { PSK, O- QPSK,ASK, } \\
\text { SPREAD SPECTRUM }\end{array}$ \\
\hline $\begin{array}{c}\text { MODESOF } \\
\text { OPERATION }\end{array}$ & $\begin{array}{c}\text { Ad-hoc/ } \\
\text { INFRASTRUCTURE }\end{array}$ & Ad-hoc & Ad-hoc \\
\hline DATA RATES & 248 Mbps & 3 Mbps & $40 \mathrm{Kbps/250} \mathrm{Kbps}$ \\
\hline TYPE OF NETWORK & LAN & PAN & PAN \\
\hline
\end{tabular}

The above table shows the comparative study of three types of network. From above table it can be clear that different types of network have different data rate, modulation and range.

\subsection{Use of AHP for Selecting Suitable Wireless Technology}

Analytical hierarchy process is basically a decision making tool, which rather than prescribing a correct decision, it help the decision maker to find one best suited for goal.

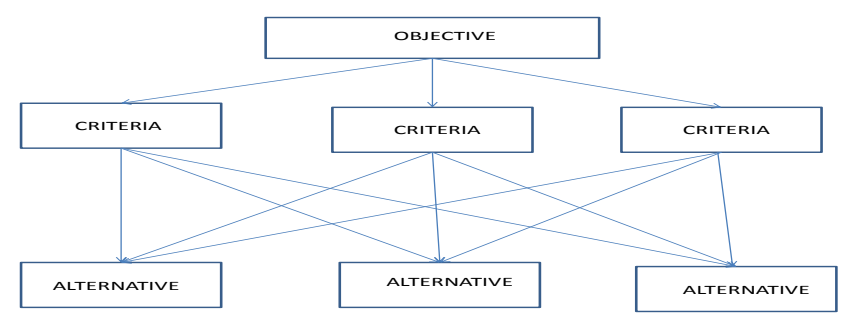

Figure 4. Basic Conceptual Diagram of AHP 
User of AHP first decomposes their decision problem into hierarchy of more easily comprehended sub-problems, each of which can be analyzed independently. Once the hierarchy is built, the decision makers systematically evaluate its various elements by comparing them to each other two at a time, with respect to their impact on an element above them in hierarchy. The AHP converts this evaluation to numerical values that can be processed and compared over the entire range of the problem.

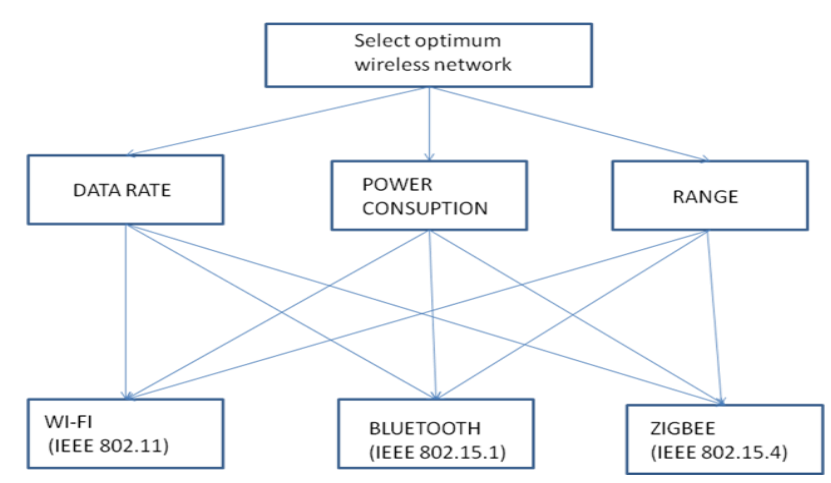

Figure 5. AHP Diagram for Wireless Technologies with Various Criteria

Data rate: $\mathrm{C}$

Power consumption: PC

Range: $\mathrm{R}$

Wi-fi: N1

Bluetooth: N2

Zigbee: N3

\section{Level 1 AHP}

Priority Value Chart

\begin{tabular}{|l|l|l|l|}
\hline & N1 & N2 & N3 \\
\hline PC & 7 & 3 & 1 \\
\hline D & 9 & 3 & 1 \\
\hline R & 3 & 1 & 9 \\
\hline
\end{tabular}

Priority Matrix

$\mathrm{A}=$

\begin{tabular}{|l|l|l|l|}
\hline & PC & D & R \\
\hline PC & 1 & 2 & 5 \\
\hline D & .5 & 1 & 3 \\
\hline R & .2 & .3 & 1 \\
\hline SUM & 1.7 & 3.3 & 9 \\
\hline
\end{tabular}

Normalisation

$\mathrm{A}^{\prime}=$\begin{tabular}{|l|l|l|l|}
\hline & PC & $\mathrm{R}$ & $\mathrm{D}$ \\
\hline $\mathrm{PC}$ & .588 & .606 & .555 \\
\hline $\mathrm{R}$ & .294 & .303 & .333 \\
\hline $\mathrm{D}$ & .117 & .090 & .111 \\
\hline
\end{tabular}




\section{Priority Vector}

$$
\begin{aligned}
& \mathrm{P}=\begin{array}{|l|l|}
\hline \mathrm{PC} & .583 \\
\cline { 2 - 3 } \mathrm{R} & .310 \\
\hline \mathrm{D} & .106 \\
\hline
\end{array} \\
& \mathrm{P}^{\prime}=\mathrm{P} \times \mathrm{A} \\
& =\begin{array}{|l|l|l|}
\hline 1 & 2 & 5 \\
\hline .5 & 1 & 3 \\
\hline .2 & .3 & 1 \\
\hline
\end{array} \times \quad \begin{array}{|c|}
\hline .583 \\
\hline .310 \\
\hline .106 \\
\hline
\end{array} \\
& =\begin{array}{|l|}
\hline .688 \\
\hline .897 \\
\hline .306 \\
\hline
\end{array} \\
& \wedge=\mathrm{P}^{\prime} / \mathrm{P}=\quad \begin{array}{|l|}
2.895 \\
\hline 2.893 \\
\hline 2.892 \\
\hline
\end{array}
\end{aligned}
$$

EIGEN VALUE $=(2.895+2.893+2.892) / 3=2.893$

$\mathrm{CI}=(2.893-3) /(3-1)=.0535$

$\mathrm{RI}=.58$ for $\mathrm{n}=3$

$\mathrm{CR}=\mathrm{CI} / \mathrm{RI}=(.0535 / .58)=.09<.1$

As CR value is less than $10 \%$, hence the above priority assignment is acceptable.

\section{Level 2 AHP}

The second level AHP matrix for deciding priority of each wireless network based on factors, i.e. data rate, power consumption and range.

\section{Priority Value Chart}

Considering Power Consumption

\begin{tabular}{|l|l|l|l|}
\hline & N1 & N2 & N3 \\
\hline PC & 7 & 3 & 1 \\
\hline D & 9 & 3 & 1 \\
\hline R & 3 & 1 & 9 \\
\hline
\end{tabular}

$\mathrm{A} 1=$\begin{tabular}{|l|l|l|l|}
\hline & $\mathrm{N} 1$ & $\mathrm{~N} 2$ & $\mathrm{~N} 3$ \\
\hline $\mathrm{N} 1$ & 1 & 2.33 & 7 \\
\hline $\mathrm{N} 2$ & .428 & 1 & 3 \\
\hline $\mathrm{N} 3$ & .142 & .33 & 1 \\
\hline $\mathrm{SUM}$ & 1.57 & 3.66 & 11 \\
\hline
\end{tabular}




\section{Normalised Value}

$\mathrm{A} 1^{\prime}=$\begin{tabular}{|l|l|l|l|}
\hline & $\mathrm{N} 1$ & $\mathrm{~N} 2$ & $\mathrm{~N} 3$ \\
\hline $\mathrm{N} 1$ & .636 & .636 & .636 \\
\hline $\mathrm{N} 2$ & .272 & .273 & .272 \\
\hline $\mathrm{N} 3$ & .090 & .090 & .090 \\
\hline
\end{tabular}

\section{Priority Vector}

$\mathrm{P} 1=$\begin{tabular}{|l|c|}
\hline $\mathrm{N} 1$ & .636 \\
\hline $\mathrm{N} 2$ & .272 \\
\hline $\mathrm{N} 3$ & .090 \\
\hline
\end{tabular}

Considering Rate

$\mathrm{A} 2=$

\begin{tabular}{|l|l|l|l|}
\hline & N1 & N2 & N3 \\
\hline N1 & 1 & 3 & .33 \\
\hline N2 & .33 & 1 & .11 \\
\hline N3 & 3 & 9 & 1 \\
\hline SUM & 4.33 & 13 & 1.44 \\
\hline
\end{tabular}

Normalised Value

$\mathrm{A} 2^{\prime}=$\begin{tabular}{|l|l|l|l|}
\hline & $\mathrm{N} 1$ & $\mathrm{~N} 2$ & $\mathrm{~N} 3$ \\
\hline $\mathrm{N} 1$ & .230 & .230 & .229 \\
\hline $\mathrm{N} 2$ & .076 & .076 & .076 \\
\hline $\mathrm{N} 3$ & .692 & .692 & .694 \\
\hline
\end{tabular}

\section{Priority Vector}

\begin{tabular}{|lr|r|}
\hline N1 & $\dot{r}$ \\
& & 230 \\
\hline & $\mathrm{N}$ & $\dot{5}$ \\
2 & & $07 \dot{6}$ \\
\hline & $\mathrm{N}$ & $\dot{2}$ \\
\hline & & $69 \dot{2}$ \\
\hline
\end{tabular}

Considering Data Rate

$\mathrm{A} 3=$

\begin{tabular}{|l|l|l|l|}
\hline & N1 & N2 & N3 \\
\hline N1 & 1 & 3 & 9 \\
\hline N2 & .33 & 1 & 3 \\
\hline N3 & .11 & .33 & 1 \\
\hline SUM & 1.44 & 4.33 & 13 \\
\hline
\end{tabular}




\section{Normalised Value}

$\mathrm{A}^{\prime}=$\begin{tabular}{|l|l|l|l|}
\hline & $\mathrm{N} 1$ & $\mathrm{~N} 2$ & $\mathrm{~N} 3$ \\
\hline $\mathrm{N} 1$ & .694 & .692 & .692 \\
\hline $\mathrm{N} 2$ & .229 & .230 & .230 \\
\hline $\mathrm{N} 3$ & .076 & .076 & .076 \\
\hline
\end{tabular}

\section{Priority Vector}

$\mathrm{P} 3=$\begin{tabular}{|l|c|}
\hline $\mathrm{N} 1$ & .692 \\
\hline $\mathrm{N} 2$ & .230 \\
\hline $\mathrm{N} 3$ & .076 \\
\hline
\end{tabular}

\section{Now, Final Priority}

\begin{tabular}{|l|l|l|}
\hline P1 & P2 & P3 \\
\hline .636 & .230 & .692 \\
\hline .272 & .076 & .230 \\
\hline .090 & .692 & .076 \\
\hline
\end{tabular}

\begin{tabular}{|l|}
\hline .583 \\
\hline .310 \\
\hline .106 \\
\hline
\end{tabular}

$=\quad$\begin{tabular}{|l|l|}
\hline $\mathrm{N} 1$ & .4646 \\
\hline $\mathrm{N} 2$ & .231 \\
\hline $\mathrm{N} 3$ & .4827 \\
\hline
\end{tabular}

Now from final priority we can conclude that N3 has highest value hence zigbee is suited for our requirement.

\section{Conclusion}

This paper deals with selection of temperature sensor for forest fire detection system. Three temperature sensors namely MLX90614, BMP085 and DS18B20 were taken into consideration. From the real time experiment it can be concluded that among these three sensors, MLX90614 is suitable for above application because of its capability to measure the temperature from distance and its faster response towards change in temperature. This paper also deals with selection of wireless network for forest fire detection system based on WSN using AHP. Three suitable networks for above application were taken into consideration namely Zigbee, Wifi and Bluetooth. Considering three network criteria namely power consumption, range and data rate, zigbee was found suitable after analyzing using AHP. Zigbee is suitable for above application because of its lower power consumption, larger range and lower data rate. As sensor node will be deployed in remote location which will mostly be battery powered, so power will be a main constraint as changing of battery of node is complex task. The node will send only the small amount of 
data hence data rate requirement of such system is low. If the range of the wireless node is higher than the number of nodes required to communicate with base station will be lower which will decrease the overall cost of system.

\section{Reference}

[1] Mohammed, S. Babani, A. Ibrahim sanka and N.A. Abdullahi, "A comparative study between different types of Temperature sensor", International Journal of Industrial Electronics and Electrical Engineering, vol. 3 , no. 12, (2015), pp. 11-13.

[2] R. Chandrasekharan, M.I. Ashiq and V. Prakash, "Forest Fire Detection using Temperature Sensors Powered by Tree and Auto Alarming using GSM", IJRSI, vol. 2, no. 3, (2015), pp. 23-28.

[3] U. Parmar and S. Singh, "Comparative study of Zigbee, Bluetooth and Wi-Fi Technology for constructing Wireless fire alarm system", International Journal of Advanced Research in Computer Science and Software Engineering, vol. 4, no. 9, (2014), pp. 893-897.

[4] Saad, B. Mostafa, E.A. Cheikh and H. Abderrahmane, "Comparative Performance Analysis of Wireless Communication Protocols for Intelligent Sensors and Their Applications", International Journal of Advanced Computer Science and Applications, vol. 5, no. 4, (2014), pp. 76-85.

[5] V. Abinayaa and A. Jayan, "Case Study on Comparison of Wireless Technologies in Industrial Applications", International Journal of Scientific and Research Publications, vol. 4, no. 2, (2014), pp. 13.

[6] A. Chauhan, R. Chauhan and S. Semwal, "Early Detection of Forest Fire Using Wireless Sensor Network", International Journal of Engineering Research and Applications (IJERA), vol. 3, no. 4, (2013), pp. 163-168.

[7] H. Kaur and S. Sharma, "A Comparative Study of Wireless Technologies: Zigbee, Bluetooth LE, Enocean, Wavenis, Insteon and UWB", Proceeding of the International Conference on Recent Trends in Computing and Communication Engineering, (2013), pp. 273-276.

[8] A. Shaikh and S. Pathan, "Research on Wireless Sensor Network Technology", International Journal of Information and Education Technology, vol. 2, no. 5, (2012), pp. 476-479.

[9] V.N. Katare, "Comparative Analysis and interpretation of various short-range wireless technologies", International Journal of Scientific \& Engineering Research, vol. 3, no. 8, (2012), pp. 1-5.

[10] R. Na, Y. Ren, Y. Hori and K. Sakurai, "Analytic Hierarchy Process aided Key Management Schemes Evaluation in Wireless Sensor Network", journal of networks, vol. 7, no. 1, (2012), pp. 88-100.

[11] T. Al-Hawari, S. Al-Bo'ol and A. Momani, "Selection of Temperature Measuring Sensors Using the Analytic Hierarchy Process", Jordan Journal of Mechanical and Industrial Engineering, vol. 5, no. 5, (2011), pp 451-459.

[12] Bhattacharyya, T. Kim and S. Pal, "A Comparative Study of Wireless Sensor Networks and Their Routing Protocols”, Sensors, (2010), pp. 10506-10523.

[13] J. Shieh, J.E. Huber, N.A. Fleck and M.F. Ashby, "The selection of Sensors", Progress in Material Science, vol. 46, (2001), pp. 461-504. 